\title{
Fundamentos para el análisis de funciones neurobiológicas: Ejecución e implementación múltiple.
}

\author{
Conceptual Underpinnings for Neurobiologial Functions: Multiple Realizability and \\ Multiple Execution.
}

\author{
Carlos Mario Muñoz-Suárez ${ }^{1 *}$ \\ 1 Grupo de Investigación Sensus. Universidad del Valle. Cali, Colombia.
}

\section{Resumen}

En este artículo se esboza una concepción general acerca de las funciones neurobiológicas. Particularmente, la manera en que se describen normativamente y las estrategias mediante las cuales se definen. Así, se clarificará, por un lado, la distinción entre implementar y ejecutar una función y, por otro, la distinción entre definir y especificar una función. Adicionalmente, se presentará un esquema detallado de cómo las funciones neurobiológicas (que pueden ser múltiplemente implementables y ejecutables) pueden ser comprendidas. Finalmente, se introducirá el concepto de "supercluster" como una unidad de modelización de funciones neurobiológicas de nivel superior.

Palabras clave: descripción para la implementación, conceptualizaciones modales y teleológicas, descripción composicional de las funciones, análisis composicional del funcionamiento, implementación y ejecución múltiple

\section{Abstract}

In this paper I will sketch a general conception about neurobiological functions. Particularly, the way in which we normatively describe them and the strategies by which we define them. So I will clarify, on the one hand, the distinction between to realize and to perform a function and, on the other hand, the distinction between to define and to specify a function. Furthermore, I will present a fine-grained scheme of how neurobiological functions (which can be multiple realizable and performable) can be grasped. Finally, I will introduce the concept of 'supercluster' as a modeling unit of higher-level neurobiological functions.

Keywords: description to realization, modal and teleological conceptualizations, compositional description of functions, compositional analysis of performing, multiple realization and performance

\footnotetext{
* Correspondencia: carlosmariomunozsuarez@gmail.com. Universidad del Valle. Cali, Colombia. Facultad de Artes Integradas, Calle 13 \# 100-00, Meléndez. Teléfono: +57 (2) 3212309

Recibido: 12-01-11. Revisión desde: 15-01-11. Aceptado: 09-05-11

DOI: $10.5839 / \mathrm{rcnp} .2011 .0601 .03$
} 


\section{Introducción}

En inteligencia artificial, neurociencia cognitiva, neuropsicología clínica y áreas asociadas se requiere una concepción básica acerca de qué son las funciones neurobiológicas. La concepción en cuestión es ontológica y su estudio se enmarca en lo que podría llamarse "metafísica de funciones": una matriz conceptual que, en el caso de las funciones neurobiológicas, pertenece al dominio de estudio de la filosofía de las neurociencias (Dennett, 1969; Bechtel et al., 2001; Northoff, 2003; Bennett \& Hacker, 2005). En este sentido, si la neurociencia cognitiva debe contar con fundamentos conceptuales para tratar con funciones neurobiológicas, necesita partir de una metafísica de funciones.

El presente trabajo tiene como objetivo general contribuir con el diseño de la concepción básica en mención, clarificando los recursos conceptuales mediante los que describimos funciones y conceptualizamos su múltiple ejecución e implementación.

\section{Definición, descripción y especificación de funciones}

Podemos definir funciones sin referirnos a las entidades que las implementan y ejecutan, e.g., 'ensamblar': unir partes sistemáticamente para formar una unidad. Asimismo, podemos describir funciones refiriéndonos a las entidades que las han de implementar y ejecutar $y$, en consecuencia, obtener descripciones para la implementación (DPI, en adelante). Definir una función, $f$, es distinto de describir $f$ para su implementación y ejecución.

Una DPI puede estructurarse con la siguiente forma general:

$D P I$ : La acción a debe o puede ser ejecutada por los objetos tipo $P$ (p1, p2... pn) e implementada en los sucesos tipo $R(\mathrm{r} 1, \mathrm{r} 2 \ldots \mathrm{rn})$ en un nivel de organización $W$.

En este sentido, las DPI para funciones neurobiológicas $(\mathrm{DP})^{\mathrm{NB}}$, en adelante) pueden estructurarse con la siguiente forma general:

$D P I^{N B}$ : La acción a debe ser ejecutada por los objetos tipo $P$ (p1, p2... pn), e implementada en los sucesos tipo $R(\mathrm{r} 1, \mathrm{r} 2 \ldots \mathrm{rn})$ en un nivel de organización del sistema nervioso $N$.

Que $a$ 'pueda ser' (i.e. 'es posible que $a$ sea') ejecutada por e implementada en ciertas entidades es distinto de que $a$ 'deba ser' ejecutada por e implementada en ciertas entidades. En el primer caso desplegamos lo que puede denominarse una conceptualización modal de $a$, mientras que en el segundo desplegamos una conceptualización teleológica de $a$. Incluir expresiones teleológicas, e.g., 'debe', en las DPI se implica del hecho de que describimos la ejecución e implementación de funciones sobre la base de pautas normativas para la atribución de propósitos a ciertas entidades o conjuntos de entidades (Millikan, 1984, 2002).

Las conceptualizaciones teleológicas presuponen lógicamente conceptualizaciones modales, no viceversa: que a deba ser implementada y ejecutada implica necesariamente que es posible que a (i.e., que a pueda ser) sea implementada y ejecutada. Por el contrario, del hecho de que sea posible que a sea implementada y ejecutada no se sigue el hecho de que $a$ deba ser implementada y ejecutada por ciertas entidades.

Las DPI incluyen identificaciones entre funciones y acciones en la medida en que intuitivamente concebimos las funciones como acciones (Millikan, 2002, p. 113). No obstante: (i) realizar una acción no implica a priori tener una función correspondiente; del hecho de que un sujeto $S$ dejó caer descuidadamente un cigarrillo encendido en un depósito de tanques de gas (acción $c$ ), no se sigue lógicamente que $S$ tenga la función de causar explosiones. (ii) No todas las acciones deben ser realizadas para ser acciones, ya que podemos concebir acciones no realizadas, e.g., triturar galaxias con un dedo índice. En este sentido, las acciones se conciben como algo que es posible realizar y no como algo que, en efecto, ha de realizarse o que estrictamente se ha realizado.

Para realizar atribuciones de funciones es indispensable realizar conceptualizaciones tanto modales como teleológicas, en la medida en que, por un lado, tenemos que tomar como punto de partida acciones posibles y, por otro, describir las acciones posibles como propósitos de entidades o conjuntos de entidades.

Las funciones neurobiológicas se implementan y ejecutan en diferentes niveles de organización del sistema nervioso. En este sentido, dos funciones pueden diferenciarse en virtud del nivel en el que tienen lugar su implementación y ejecución; e.g., podemos atribuir funciones a regiones de la corteza cerebral y, también, a partes de regiones de la corteza cerebral, diferenciando funciones mediante una distinción de constitución entre partes de un sistema.

Si no son identificadas las entidades en las que se implementa y por las que se ejecuta una función $f$, entonces $f$ no podrá ser especificada en el nivel adecuado $y$, por constitución, tampoco en el sistema. Si $f$ no es especificada, i.e. si $f$, en tanto que acción posible, no es descrita como un propósito de entidades en un nivel particular, no obtendremos una DPI ${ }^{\mathrm{NB}}$. En este sentido, las DPI ${ }^{\mathrm{NB}}$ han de incluir expresiones que se refieran a niveles de organización del sistema nervioso. $\mathrm{Si}$ al describir $f$ no se especifican los objetos y sucesos que la ejecutan e implementan (respectivamente) y, por tanto, ciertos niveles de organización, entonces no tendremos una DPI ${ }^{\mathrm{NB}}$.

De acuerdo con la conceptualización modal, una función puede ser múltiplemente implementada y ejecutada por entidades de distinto tipo y nivel. Los conceptos de 'múltiple implementación' y de 'múltiple ejecución' de una función $f$ tienen sentido en la medida en que podemos definirlos sin especificarlos, es decir, sin atribuir ejecutores ni implementadores a $f$. Esto se debe en gran medida a que los términos con los que designamos funciones no designan, por sí mismos, acciones implementadas y ejecutadas. Especificar una función implica identificar el conjunto de entidades que la pueden o deben ejecutar e implementar. En consecuencia, $f$ puede ser especificada en diferentes niveles de organización bajo una misma definición. Así pues, podemos ofrecer explicaciones funcionales de un sistema sin incluir descripciones físicas del mismo. En otras palabras, podemos definir las acciones que pueden tener lugar en un sistema sin especificarlas (esto permea la distinción entre teorías computacionales de la cognición y teorías neurobiológicas de la cognición). 
Alternativamente, si $f$ es definida como una función neurobiológica, entonces su definición tendrá que incluir conceptualizaciones teleológicas acerca de entidades neurobiológicas que desempeñen los papeles de ejecutores e implementadores.

Las DPI fijan pautas normativas para que ciertas acciones tengan lugar pero, por sí mismas, no conllevan el hecho de que esas pautas se cumplan. Así pues, 'acción', dentro de las DPI, no refiere a una acción que esté teniendo lugar sino, más bien, a una acción que posiblemente tendrá lugar o que debería tener lugar, i.e. a un suceso que posiblemente será o debería ser una parte temporal de un sistema si y solo si tiene lugar en el mismo. Esta postura implica la aceptación de que las acciones son sucesos que pueden o no ser partes temporales de los sistemas en los que tienen lugar.

En este sentido, los sucesos, y por consiguiente las acciones, tienen lugar en el tránsito entre un estado de cosas y otro (Davidson, 1970). En este punto es pertinente introducir algunas puntualizaciones acerca de la identidad de los sucesos (para ampliar: Kim, 1976; Lombard, 1979; MacDonald, 2005, cap. 5). Tomemos el objeto $a$ y las propiedades $Q$ y $F$. Las entidades $a$ y $Q$ forman un estado de cosas $Q a$, en $t 1$, que pasa a ser un estado de cosas $F a$, en $t 2$. Entre $t 1$ y $t 2$ tuvo lugar un cambio, tanto en las propiedades que instancia a como en los objetos en los que se instancian los ejemplares de propiedades $Q$ y $F$ instante tras instante. Si el tránsito entre $Q a$ y $F a$ es un suceso $f$, entonces, tanto la propiedad que tiene $a$ de instanciar $Q$ y $F$ en función del tiempo, como las propiedades que tienen $Q$ y $F$ de estar instanciadas en $a$ en función del tiempo son condición de posibilidad para $f$; pero $f$, en sí mismo, no es ni un objeto, ni una propiedad, ni un estado de cosas.

Parece razonable aceptar que al hablar de sucesos capturamos referencialmente cambios en estados de cosas ya que, prima facie, desplegamos dos criterios ontológicos: (1) los sucesos necesariamente tienen partes temporales: iniciales, medias y finales, y (2) los sucesos contingentemente tienen partes espaciales: el cambio $Q a_{t 1}-F a_{t 2}$ es posiblemente distinto del cambio $Q a_{t 1}-F a_{t 2}$ si $a, Q$ y $F$ de $t 1$ a $t 2$ en el primer caso ocupan una región de espacio distinta a la que ocupan en el segundo caso. Ni (1) ni (2) son suficientes para distinguir un suceso de otro. Adicionalmente, un suceso, e.g., $f$ (un aumento de polaridad negativa en la unidad $a$ entre $t 1$ y $t 3$ ) se da solo si $a$ instancia la propiedad $P$ (<aumentar la polaridad negativa $>$ ) en dicho periodo. Por supuesto, todos los estados de polaridad, instante por instante entre $t 1$ y $t 3$, son el conjunto total de propiedades que $a$ instanciará en ese periodo en la medida en que instancia $P$. Así, $P$ es condición de posibilidad para la instanciación de otras propiedades en $a$. Si $a$ no instancia $P$, entonces tampoco tendrá más de un estado de polaridad negativa mayor al que ya tiene. En este sentido, un suceso conlleva la instanciación periódica de propiedades por parte de los objetos que involucra.

Volviendo al tema central, una función $f$, a pesar de ser especificada en el sistema nervioso central, e.g., puede ser definida mediante el uso de una matriz constituida por relaciones nomológicas entre salidas e insumos, sin incluir alusiones a estados neurobiológicos (Putnam, 1967). Así definida, $f$ no es descrita como una función propiamente neurobiológica, ya que puede ser ejecutada e implementada por entidades inorgánicas.
La diferencia entre 'definir una función' y 'especificar una función en un sistema' nos permite comprender por qué la neuroanatomía, la neuroquímica, la neurofisiología y la neuropsicología, entre otras, intentan explicar las mismas funciones (e.g., el control del ciclo circadiano) recurriendo al análisis de diferentes niveles de organización, es decir, identificando funciones mediante la especificación de las mismas en ciertos objetos y sucesos (e.g., células, moléculas, patrones de actividad eléctricos, cambios metabólicos en regiones corticales, comportamientos específicos frente a ciertos insumos etc.).

En el momento en que ofrecemos una $\mathrm{DPI}^{\mathrm{NB}}$ para una función $f$, especificamos también el conjunto de entidades neurobiológicas en las debe estar implementada y por las que debe ser ejecutada, y no meramente aquellas entidades, neurobiológicas o no, en las que puede estar implementada y por las que puede ser ejecutada. En este sentido, una función neurobiológica es descrita como un propósito de (algo que deben implementar y ejecutar) ciertas entidades neurobiológicas.

Las funciones más generales atribuidas al sistema nervioso (e.g., el aprendizaje de ciertos patrones motores), al ser meramente definidas, son funciones que pueden ser múltiplemente implementadas, tanto en sistemas orgánicos como inorgánicos (Putnam, 1967; Fodor, 1974; Dennett, 1971). Así, si éstas funciones merecen ser calificadas de 'neurobiológicas' será solo en la medida en que sean especificadas en entidades postuladas por teorías neuro-biológicas. Si afirmamos que una función neurobiológica puede ser ejecutada e implementada en entidades inorgánicas de tipo $C$, entonces deberá poder ser definida independientemente de su especificación en entidades orgánicas. Una función $f$, que puede ser múltiplemente especificada, puede lógicamente ser múltiplemente implementada; sin embargo, si $f$ es definida como una función neurobiológica, entonces tendrá que ser concebida y descrita como una función que debe ser ejecutada por e implementada en entidades neurobiológicas. Responder al «por qué» una función neurobiológica $f^{*}$ debe ser ejecutada por e implementada en entidades de un nivel $K$ (i.e., por qué $f^{*}$ ha de ser descrita como un propósito de entidades en $K$ ) requiere del desarrollo de una postura acerca de la historia $K$ que nos permite relacionar, mediante una conceptualización teleológica, $f^{*}$ con $K$, e.g., los neoteleo-logistas justifican la atribución de pautas normativas sobre la base de procesos de selección natural (Millikan, 1984; Neander, 1991; Wimsatt, 2002). Al realizar este proceso, obtendremos también una justificación empírica de la pauta normativa correspondiente.

El problema de la justificación de la atribución de pautas normativas es el problema de por qué las entidades de un tipo particular deben implementar y ejecutar una función determinada. Permaneceré neutral respecto de este problema, a pesar de que esboce algunas tesis relevantes para el mismo. Este problema se relaciona directamente con la ingeniería inversa, que trata de averiguar la función para la que una máquina fue diseñada (Pinker, 1998, p. 21 y ss.). Una función puede ser (de acuerdo con su definición) implementada en un conjunto de niveles de organización que incluya los niveles $\phi 1$, $\varphi 2 \ldots \varphi n$, que, a su vez, incluyan tanto entidades orgánicas como inorgánicas. Lo anterior puede denominarse implementación múltiple híbrida. 


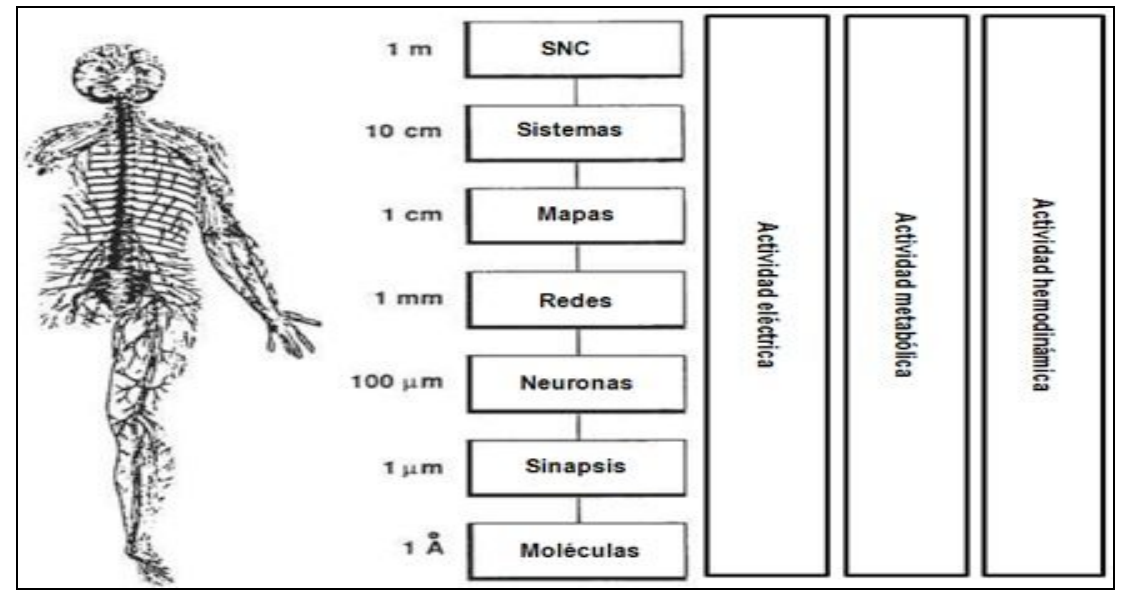

Esquema 1. Modificado de [Sejnowski \& Churchland (1996), p. 9].

$\mathrm{Si}$ bien las funciones son concebidas como acciones posibles o actuales -y estas últimas, a su vez, como sucesos-, las funciones no necesariamente deben ser concebidas como sucesos que tienen o han tenido lugar. El funcionamiento de un conjunto de entidades es diferente de las funciones que se atribuyen a este. Así, el funcionamiento puede ser definido como una función implementada y ejecutada, es decir, como aquello que ejemplifica una DPI, e.g., si se alteran los sucesos en un sistema $J$, entonces se alterará el funcionamiento de $J$ : esta alteración en el funcionamiento no implica, por sí mismo, la falsedad de la atribución de la pauta normativa correspondiente, de modo que la atribución de la función permanece, a pesar de que existan fallas de funcionamiento. Millikan ha defendido esta tesis sin la alusión explicita a la distinción que presento entre funcionamiento y función (Millikan, 2002, p. 116). Para una postura crítica, vid.: Davies (2001).

La distinción mencionada motiva la aceptación de que un sistema con un funcionamiento anómalo pueda recuperar su funcionamiento normal (Neander, 1991), ya que continúa siendo objeto de atribución de una pauta normativa $y$, específicamente, de una DPI. Así, la distinción entre funcionamiento y función da sentido a la noción de restauración funcional (Saab, 2007, p. 145 y ss.), una noción central, e.g., para los estudios en neuropsicología clínica y en biónica.

El funcionamiento de un sistema (es decir, las acciones que tienen lugar en él) puede comprenderse sobre la base de un análisis de las capacidades que tienen las entidades que lo constituyen. Por consiguiente, el malfuncionamiento puede explicarse en términos de cambios anómalos en las entidades constitutivas, e.g., la epilepsia es usualmente explicada en términos de anomalías en sucesos cerebrales eléctricos. En este sentido, podemos construir explicaciones del malfuncionamiento mediante un análisis funcional (Cummins, 1975) multinivel de las anomalías, de modo que si el daño en el sistema es severo, la función no podrá volver a ser ni implementada ni ejecutada, i.e., no podrá darse cierto funcionamiento, a pesar de que podremos continuar realizando conceptualizaciones teleológicas sobre dicho sistema. El hecho de que un sistema $d$ pierda su funcionamiento no implica, lógicamente, que $d$ pierda una función, sino más bien que es un caso de malfuncionamiento. Ahora bien, desde mi punto de vista, por un lado, que un sistema $d$ sea acreedor de ciertas funciones depende del hecho de que podamos construir DPI para $d y$, por otro, que $d$ tenga ciertos funcionamientos depende del hecho de que $d$ o partes de $d$ puedan tener ciertas propiedades, e.g., <cambiar la polaridad positiva>. Comprender las condiciones para el funcionamiento de un conjunto de objetos puede ayudarnos a identificar funciones, es decir, a construir pautas normativas funcionales y a postular DPI.

\section{Niveles, múltiple implementación y múltiple ejecución de funciones neurobiológicas}

En la anatomía del sistema nervioso se diferencian niveles de organización en virtud de órdenes de magnitud, i.e., $1 \AA \AA, 1 \mu \mathrm{m}$, $100 \mu \mathrm{m}$ etc., o de niveles de observación de objetos materiales. Las funciones neurobiológicas, de acuerdo con un conjunto de $\mathrm{DPI}^{\mathrm{NB}}$, deben ser ejecutadas por objetos presentes en alguno de estos niveles de organización. Cada nivel de organización tiene sus propios objetos elementales diferenciados por órdenes de magnitud, e.g., moléculas.

Algunas funciones neurobiológicas implementadas y ejecutadas en los niveles de organización molecular, sináptico, celular, etc., suelen ser descritas, inicialmente, en la medida en que sean conocidos tales objetos y sus sucesos asociados. En otras palabras, estas funciones suelen ser especificadas al ser descritas e identificado cierto funcionamiento. Complementariamente, aquellas funciones neurobiológicas implementadas y ejecutadas por niveles de organización de redes, mapas, sistemas etc., suelen ser descritas, inicialmente, con independencia de los objetos y sucesos de un nivel de organización neurobiológico particular (modo de definición que motiva la categorización de estas funciones como superiores). Esta distinción permite identificar dos estrategias:

1.Estrategia de descripción de funciones neurobiológicas abajo-arriba (EBU): la acción a es una función neurobiológica $f$, ya que debe ser ejecutada por los objetos tipo $P$ (p1, p2... pn) e implementada en los sucesos tipo $R(r 1, r 2 \ldots r n)$ en el nivel de organización $N$ del sistema nervioso. 
2.Estrategia de descripción de funciones neurobiológicas arriba-abajo (EUD): la acción a es una función neurobiológica $f$, ya que puede ser ejecutada por objetos de algún tipo e implementada en los sucesos de algún tipo en algún nivel de organización del sistema nervioso.

Como se mencionó, EBU supone lógicamente EUD pero no a la inversa. Con EBU describimos funciones neurobiológicas de nivel inferior y con EUD describimos funciones de nivel superior (algunas de las cuales denominamos también funciones cognitivas). La EUD no es una estrategia de descripción de funciones estrictamente neurobiológicas, sino de funciones que pueden ser neurobiológicamente implementadas.

A medida que ascendemos por los niveles de organización, la relación entre objetos y funciones se hace semánticamente menos estrecha, ya que, e.g., la descripción de funciones atribuidas a entidades al nivel de organización de $10 \mathrm{~mm}$ no tiene que incluir ni nombres ni descripciones que refieran a entidades en el orden de $10 \mathrm{~mm}$, e.g., procesar información cromática. Por lo general, las funciones neurobiológicas atribuidas a entidades de niveles de organización moleculares (i.e., del orden de $1 \AA ̊$ ) incluyen en su descripción nombres para dichas entidades (Churchland, 1989, p. 110). Así, funciones como el procesamiento de información cromática deben ser funciones neurobiológicas que pueden ser ejecutadas por e implementadas en entidades de múltiples niveles de organización.

El panorama debe ser complementado con niveles de actividad definidos en relación con los tipos de sucesos involucrados. La motivación para incluir niveles de actividad ejemplifica la idea de que un conjunto de sucesos puede hacer parte de otro e incluir otro. Esto implica que los sucesos de un nivel dependen nomológicamente de sucesos que tienen lugar en un nivel de actividad inferior $y$, quizás, superior (ver esquema 1).

La distinción entre niveles de actividad y niveles de organización corresponde con la distinción entre ejecutar e implementar: una función es ejecutada por un conjunto determinado de objetos de un nivel de organización y es implementada en un conjunto de sucesos en un nivel de actividad. Así, si contamos con una DPI ${ }^{\mathrm{NB}}$, contamos con especificaciones no solo en niveles de organización sino, también, en niveles de actividad, e.g., los sucesos eléctricos al nivel de $1 \mathrm{~mm}$ son distintos de los sucesos eléctricos al nivel $1 \mathrm{~cm}$, lo cual indica que sin diferencia de tipo hay diferencia de escala. Así, muchos sucesos eléctricos, e.g., oscilaciones de voltaje en membranas celulares, son explicados con base en otros sucesos que tienen lugar en niveles inferiores, e.g., cambios iónicos en proteínas de membranas (Llinás, 2001, p. 10 y ss.). En consecuencia, los medios para el registro de diferentes tipos de sucesos son diferentes, e.g., SPECT, PET, fMRI, etc. (Buckner \& Logan, 2006, p. 28).

Contamos con evidencia de casos en los que el funcionamiento se conserva, a pesar de que parte de las entidades que deberían ejecutar e implementar la función correspondiente estén alteradas o destruidas: tanto los niveles de organización como de actividad no solo guardan cierta independencia explicativa, sino también cierta independencia causal, i.e., no hay una relación estrictamente necesaria entre cierto funcionamiento y un conjunto determinado de objetos. La probabilidad deriva, principalmente, de la plasticidad neuronal (Brown \& Sherrington, 1912; Kaas, 2000; Recanzone, 2000): la propiedad del sistema nervioso para implementar y ejecutar ciertas funciones en un rango determinado de sectores. Tanto la plasticidad cerebral y los casos en los que el sistema nervioso realiza restauraciones funcionales automáticas representan evidencia empírica a favor de la tesis de la implementación y ejecución múltiple de las funciones. Así pues, una $D P I^{N B}$ es una descripción probabilística.

Arribamos a la siguiente definición: Implementación múltiple y ejecución múltiple de las funciones neurobiológicas (IEMB): la acción $a$ es una función múltiplemente implementable y múltiplemente ejecutable si puede ser descrita mediante una conceptualización teleológica acerca de entidades neurobiológicas pero, a su vez, no bloquea la posibilidad de conceptualizaciones modales que incluyan objetos y sucesos de distinto tipo a los referidos mediante la conceptualización teleológica.

\section{Análisis composicional del funcionamiento}

Introducir niveles de actividad abre la posibilidad a un análisis composicional del funcionamiento. Si contamos con una DPI ${ }^{\mathrm{NB}}$ para la función $a$, entonces $a$ puede ser explicada mediante un conjunto de DPI ${ }^{\mathrm{NB}}$ acerca de funciones sub-a1, sub-b2... sub-n, en donde $a$, por especificación, debe ser implementada en y ejecutada por entidades de un nivel de organización compuesto por las entidades en las que deben implementarse y por las que deben ejecutarse las funciones sub-a1, sub-b2... sub-n.

En este caso, no solo estaremos realizando un análisis relacional de funciones neurobiológicas, sino que también podremos realizar un análisis composicional del funcionamiento. Una vez especificada una función, esta puede ser redescrita. Así, si definimos a como composicionalmente dependiente de las funciones sub-a1, sub-b2... sub-n, podremos obtener una descripción composicional de $a$. De este modo, una descripción composicional permite especificar ciertas relaciones que una función puede o debe establecer con otras. La forma general de una descripción composicional del funcionamiento (DCF) puede ser presentada así:

DCF: La función a puede ser ejecutada por los objetos tipo $P$ (p1, p2... pn) e implementada en los sucesos tipo $R(r 1, r 2 \ldots r n)$ en un nivel de organización $N$, si una función o conjunto de funciones sub-a puede(n) ser ejecutada(s) por los objetos tipo sub $P$ (sub p1, sub p2... sub pn) e implementada(s) en los sucesos tipo sub $R$ (sub $r 1$, sub $r 2 \ldots$ sub $r n$ ) en un nivel de organización constitutivo de $N, N-1$.

No todo análisis relacional de funciones conduce a una DCF, ya que no todos los análisis relacionales son análisis composicionales, a pesar de que todos los análisis composicionales son análisis relacionales. En general, las DCF son fundamentales para una comprensión sistémica funcional. Las DCF permiten vislumbrar la posibilidad de que la atribución de una función sea comprendida en términos de funcionamientos que tienen lugar en diferentes niveles de un mismo sistema. Así, la evolución conjunta de estudios a múltiple 


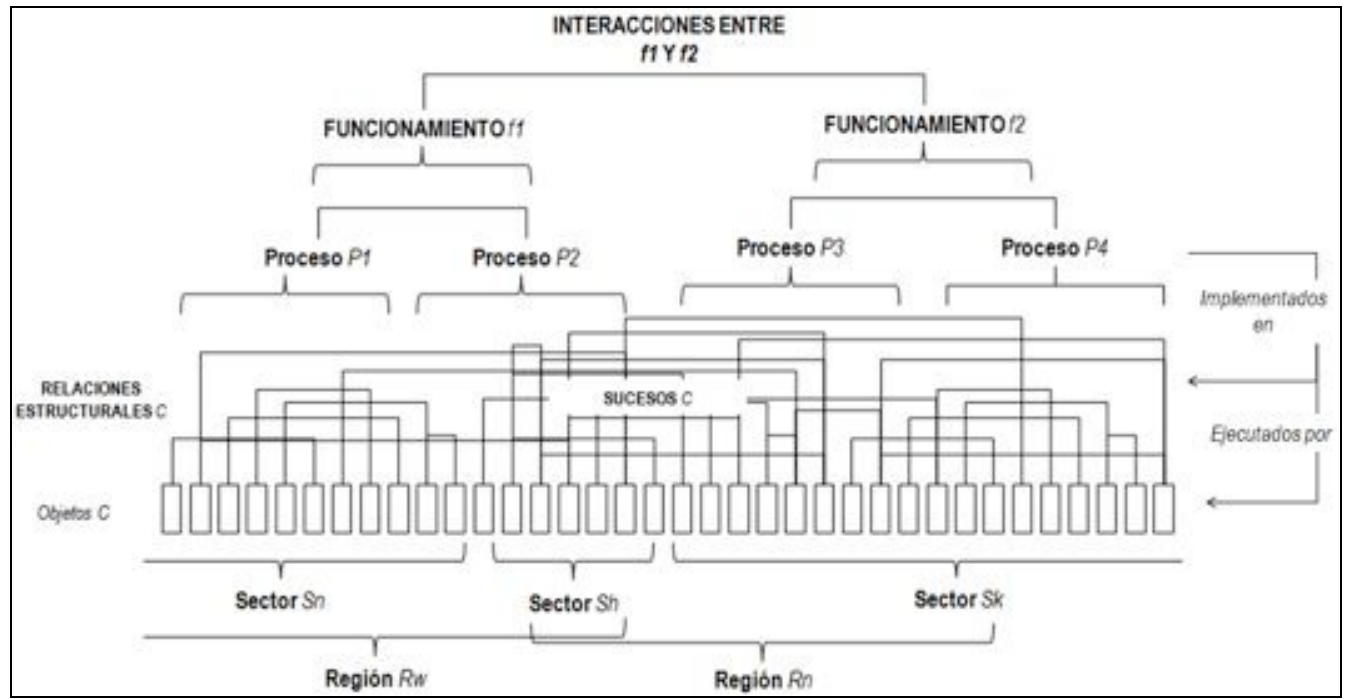

Esquema 2.

nivel, e.g., fisiológicos, comportamentales, neuroimagenológicos funcionales, etc., puede conllevar a la comprensión de una función. Lo anterior justifica el estudio interdisciplinar de las funciones neurobiológicas e, indirectamente, fortalece la tesis de que los términos con los que designamos funciones superiores atribuidas a sistemas neurobiológicos no son nombres de clases naturales.

Usualmente se afirma que si un sistema, debido a su constitución física, no está en disposición de hacer algo (i.e., no tiene la capacidad de hacer algo), entonces no ha perdido una función o propósito para el que fue diseñado. No obstante, las funciones, por definición, no son capacidades. Un sistema tiene una capacidad si está en disposición de hacer algo y, adicionalmente, si dicho sistema está compuesto por entidades en las que (de facto) pueden tener lugar cierto tipo de sucesos. Si un sistema $S$ no esta en disposición de hacer algo que debería hacer, entonces $S$ ha perdido la capacidad de hacerlo pero no necesariamente la función descrita sobre la base de una conceptualización teleológica. Así, un sistema tiene una capacidad si (de facto) puede tener cierto(s) funcionamiento(s). Lo cual no ocurre para el caso de las funciones. Esta distinción permite clarificar el concepto de restauración funcional en términos de restauración de capacidades. Si, estrictamente, afirmamos que un sistema ha perdido una función (conceptualizada teleológicamente) tendremos que afirmar que dicho sistema no debería hacer algo y, por consiguiente, desmotivar la recuperación del funcionamiento.

Explicar lo que un sistema está en disposición de hacer implica un análisis de las condiciones causales para que tenga cierto funcionamiento, lo que, a su vez, puede implicar DCF. EI análisis de las capacidades de un sistema permite, en cierto sentido, justificar la atribución de funciones, e.g., si $S$ (sin ser un caso anómalo) no es capaz de percibir colores, entonces, $\mathrm{S}$ no ha de concebirse como algo que deba percibir colores; a pesar de que, mediante una conceptualización modal, admitamos que es posible que $S$ perciba colores.

Adicionalmente, las DCF permiten establecer vínculos entre capacidades. Así, dado el caso en que un sistema $S$ presente un desempeño anómalo debido a una falla en una parte $g$, entonces, quizás, otra parte de $S$ pueda suplir el malfuncionamiento. Lo anterior implicaría que $S$ está en disposición y tiene la capacidad de compensar el malfuncionamiento de $g$. El concepto de restauración de capacidades se encuentra, en este sentido, relacionado con el concepto de disposición para actuar y, por consiguiente, con la atribución de capacidades y de condiciones para el funcionamiento.

\section{Síntesis y conceptos adicionales}

Partamos del siguiente principio epistemológico: el conocimiento de un conjunto de objetos físicos no implica a priori el conocimiento de todos los sucesos que los incluyen. Esto, e.g., explica por qué podemos diferenciar los explananda de la neuroanatomía de los explananda de la neurofisiología. Los objetos en un nivel determinado tienen relaciones estructurales y estas, a su vez, tienen relaciones nomológicas (contingentes) con ciertos sucesos.

Una función no se encuentra implementada en relaciones estructurales (conexiones físicas) sino en la actividad que depende de dichas relaciones estructurales, i.e., se encuentra implementada en cierto conjunto de sucesos. Por consiguiente, la presencia de ciertas relaciones estructurales no es condición suficiente (aunque sí nomológicamente necesaria) para el funcionamiento.

Podemos identificar, por un lado, relaciones estructurales $y$, por otro, relaciones entre funcionamientos. Lo primero no conlleva por sí mismo lo segundo, ni viceversa. Pensar, e.g., que el análisis de las relaciones entre funcionamientos conlleva por sí mismo el análisis de relaciones estructurales conduce al localizacionismo. Como bien afirman Kosslyn y Anderson:

"[...] the mistake of the early localizationists is that they tried to map behaviors and perceptions into single localizations in the cortex. [Por el contrario] [... a]ny particular 
behavior or percept is produced by many areas, located in various parts of the brain. Thus [...] complex functions such as perception, memory, reasoning, and movement are accomplished by a host of underlying processes, each of which confers only a single facet of the ability [...] they do not "think" but rather reflexively perform a specific operation when provided with appropriate input. It is these simple, underlying processes that are carried out in a single region of the brain. Indeed, the abilities themselves typical can be accomplished in numerous different ways, which involve different combinations of processes [...] Any given complex ability, then is not, accomplished by a single part of the brain [...] However, simple processes that are recruited to exercise such abilities are localized." (Kosslyn \& Anderson, 1992, p. XIX. )

Tres términos técnicos pueden ser introducidos: 'áreas', 'regiones' y 'sectores'. Adoptaré las siguientes definiciones: un área es una parte de la corteza cerebral citoarquitectónicamente delimitada (Brodman, 1907); una región es una parte de la corteza cerebral identificada con base en su localización relativa (izquierdo, derecho, ventral, medial, dorsal, lateral, anterior, posterior, etc.) y en las divisiones generales de los lóbulos (e.g., giro frontal medio, inferotemporal, parieto-temporo-occipital anterior, polo temporal, etc.); finalmente, un sector es una parte de cualquier región y no solo de aquellas identificadas en la corteza a partir de diferencias observacionales explícitas (i.e., giros y fisuras).

En la medida en que una función es concebida como una acción posible o actual, depende de microacciones que la constituyan. Estas microacciones pueden denominarse 'procesos'. En este sentido, una función depende de un conjunto de procesos. Estos no pueden ser concebidos como etapas linealmente organizadas, sino más bien como fases funcionales que pueden implementarse en paralelo.

Asimismo, un funcionamiento depende de una cadena de sucesos en los que se implementa una función. Cierto conjunto de procesos refleja cierta fase de una función y así contribuye con el funcionamiento total que identifica la implementación y ejecución de la función, e.g., una función $f$ puede depender de los procesos $f_{1}, f_{2} \ldots f_{n}$. Así, $f$ puede ser ejecutada por las estructuras $c$, en cuyas relaciones estructurales se dan sucesos en los que se implementan las fases $f_{1}, f_{2} \ldots f_{n}$, dando lugar a los procesos correspondientes.

Una DPINB de $f$ puede incluir una descripción de las fases o procesos de los que depende $f$ y, asimismo, una especificación de procesos. No podemos realizar una especificación de grano fino de la función $f$ si no hemos especificado los procesos mediante los cuales tiene lugar el funcionamiento correspondiente.

Así, una función neurobiológica está implementada en un conjunto de sucesos que, a su vez, constituyen procesos. Partiendo de una DPI ${ }^{\mathrm{NB}}$ detallada, el conjunto total de sucesos implementando procesos es aquello en lo que se encuentra implementada una función particular. A este conjunto de sucesos denominaré 'supercluster'. Los procesos, desde este punto de vista, pueden ser explicados en términos de subfunciones, de modo que la conexión dinámica entre superclusters puede ser analizada con base en DCF.

Las funciones mismas, en tanto que acciones actuales, se encuentran implementadas en los sucesos que implementan procesos. Cualquier modelo computacional de una función y de los procesos de los que depende implica la caracterización de las unidades de modelización de la función neurobiológica. Proponer un tipo de unidad de modelización de funciones neurobiológicas depende del nivel de registro del recurso tecnológico empleado. Propongo los superclusters como unidades de modelización de funciones definidas con la EUD y descritas con $\mathrm{DPI}^{\mathrm{NB}}$ y DCF. De este modo, la especificación de superclusters depende del registro de ciertos conjuntos de sucesos que implementen procesos para el desempeño de una función particular, aun en casos de restauración automática o propiciada de capacidades por causas externas al sistema. Cómo realizar modelizaciones computacionales mediante la identificación de superclusters es un análisis que tendrá que ser presentado en otro trabajo.

\section{Referencias}

Bechtel, W., Mandik, P. \& Mundale, J. (2001). Philosophy meets the neurosciences. En: Bechtel, W., Mandik, P., Mundale, J. \& Stufflebeam, R. (2001), Philosophy and the Neurosciences, (pp. 423). Oxford: Blackwell Publishers.

Bennett, M. \& Hacker, P. (2005). Philosophical Foundations of Neuroscience. Pdstow: Blackwell.

Brodman, K. (1907). Beiträge zur histologischen Lokalisation der Großhirnrinde. Journal fur Psychologie und Neurologie, 10, 231234.

Browm, G. \& Sherrington, C. (1912). On the instability of a cortical point. Proceedings of Royal Society of London B, 85, 250- 277.

Buckner, R. \& Logan, J. (2006). Functional Neuroimaging Methods: PET and fMRI. In Cabeza, R. \& Kingstone, A. (comps.). Handbook of Functional Neuroimaging and Cognition (pp. 27-48). Bradford Books.

Churchland, P. \& Sejnowski, T. (1996). The Computational Brain. Cambridge: MIT Press.

Churchland, P.M. (1989). A Neurocomputational Perspective. Cambridge, M. A.: The MIT Press.

Cummins, R. (1975). Functional Analysis. The journal of Philosophy, 72(20), 741- 765.

Davidson, D. (1970). Events as Particulars. Nôus, 4, 25- 32.

Davies, P. (2001). Norms of Nature: Naturalism and the nature of Functions. Cambridge, Mass.: The MIT Press.

Dennett, D. (1969). Content and Consciousness. Londres, Routledge and Kegan Paul.

Dennett, D. (1971). Intentional Systems. Journal of Philosophy, 68, 87106.

Fodor, J. (1974). Special Sciences: Or the Disunity of Science as a Working Hypothesis. Synthese, 28, 97- 115.

Kaas, J. (2000). The Reorganization of Sensory and Motor Maps after Injury in Adult. In Gazzaniga, M. (Eds.), The New Cognitive Neuroscience (pp. 223-236). Cambridge, Mass, London: MIT Press.

Kim, J. (1976). Events as Property Exemplifications. In Brand \& Walton (Eds.), Action Theory (pp. 159-177). Dordrecht, Reidel.

Kossly, S. \& Anderson, R. (1992). Frontiers in Cognitive Neuroscience. Cambridge: MIT Press.

Llinás, R. (2001). I of the Vortex. From Neurons to Self. Cambridge: MIT Press.

Lombard, L. (1979). Events' Canadian. Journal of Philosophy, 9, 425460.

MacDonald, C. (2005). Varieties of Things. Blackwell Publishing. 
Millikan, R. (1984). Language, Thought and other Biological Categories. Cambridge: MIT Press.

Millikan, R. (2002). Biofunctions: Two Paradigms. In Ariew, A. Cummins, R. \& Perlman, M. (Eds.), Functions (pp. 113-143). New York: Oxford University Press.

Neander, K. (1991). Functions as Selected Effects: The Computational Analyst's Defense. Philosophy of Science, 58, 168-184.

Northoff, G. (2003). Philosophy of the Brain: The Brain Problem, Amsterdam/Philadelphia, John Benjamins Publishing Company.

Pinker, S. (1998). How the Mind Works. London: Penguin Books.

Putnam, H. (1967). Psychological Predicates. In Captin \& Merrill (Eds.), Art, Mind, and Religion (pp. 37-48). Pittsburg: University of Pittsburg Press.

Recanzone, G. (2000). Cerebral Cortical Plasticity: Perception and Skill Acquisition. In Gazzaniga, M. (Ed.), The New Cognitive Neuroscience (pp. 237-247). Cambridge, Mass, London: MIT Press.

Saab, S. (2007). Los senderos de la explicación mental. México DF: Instituto de Investigaciones filosóficas.

Wimsatt, W. (2002). Functional Organization, Analogy, and Inference. In Ariew, A. Cummins, R. \& Perlman, M. (Eds.), Functions (pp. 173221). New York: Oxford University Press. 